at one year after diagnosis. We found that a significant improvement was achieved across all the different outcome measures from the first month of treatment in a good proportion of patients. The rate of improvement was slower after the first month and the maximum improvement was seen at six months, however, very little or no change at one year.

\section{G459(P) ABSTRACT WITHDRAWN}

\section{G460(P) INTERVENTIONS FOR AUTUMN EXACERBATIONS OF ASTHMA IN CHILDREN: A SYSTEMATIC REVIEW}

${ }^{1} \mathrm{M}$ Akhbari, ${ }^{2} \mathrm{D}$ Kneale, ${ }^{3} \mathrm{KM}$ Harris, ${ }^{4} \mathrm{KC}$ Pike. ${ }^{1}$ The GKT School of Medical Education, King's College London, London, UK; ${ }^{2}$ EPPI-Centre, Social Science Research Unit, UCL Institute of Education, University College London, London, UK; ${ }^{3}$ Centre for Paediatrics, Blizard Institute, Queen Mary University of London, London, UK; ${ }^{4}$ Respiratory, Critical Care and Anaesthesia, UCL Great Ormond Street Institute of Child Health, London, UK

\subsection{6/archdischild-2018-rcpch.448}

Aims Asthma exacerbations peak in school-aged children following the autumn school return. Modifiable factors, including poor treatment adherence during the summer months and increased allergen and viral exposure may underlie these observations. Interventions implemented in anticipation of the autumn school return might lessen the burden upon patients and healthcare resources. We undertook a Cochrane systematic review to assess the effectiveness of interventions aiming to reduce asthma exacerbations in children returning to school.

Methods Randomised controlled trials (RCTs) were identified in searches of the Cochrane Airways Group Specialised Register (CAGR) and other supplementary sources. Eligibility criteria included study design (RCTs), intervention (intended to reduce autumn exacerbations) and population (participants aged 18 years or younger). We appraised the quality of trials using the Cochrane Risk of Bias tool.

Results 520 records were retrieved. 4 studies met the inclusion criteria and together randomised 14048 children to receive an intervention or usual care. Two studies employed the leukotriene receptor antagonist (LTRA) montelukast, one used omalizumab or an inhaled corticosteroids boost and the last analysed the impact of a reminder letter sent to caregivers from Primary Care Providers about asthma medication. Quality ratings in most domains were low risk.

Risk of exacerbation were significantly reduced in single studies of omalizumab OR 0.48 (95\% CI: 0.25 to 0.92 ) and montelukast OR $0.25(0.08-0.79)$. Frequency of unscheduled contacts increased during September (OR 1.30 95\% CI: 1.03 to 1.66$)$ due to the reminder correspondence. The incidence of adverse events did not differ between trial arms. An updated search retrieved 25 further studies, including a randomised open trial of pranlukast. This un-blinded study was of poorer quality, relying largely upon subjective outcomes. Pranlukast was found to be superior to usual care in reducing worsening asthma symptoms only in boys aged 1-5 years.

Conclusion Omalizumab treatment initiated 4-6 weeks in advance of the school return might reduce autumn asthma exacerbations. This review identified a need for coordinated research employing validated measurement tools to explore patient-important outcomes including the impact of interventions on asthma control or quality of life. We would recommend that exacerbation definition be standardised in future trials.

\section{G461(P) LUNG FUNCTION MEASUREMENT FOLLOWING SURGERY IN IDIOPATHIC SCOLIOSIS}

IE Williams, E Finnerty, M Rutter, M Khan, S Charlton, R Ross Russell. Paediatric Respiratory, Addenbrooke's Hospital, Cambridge, UK

\subsection{6/archdischild-2018-rcpch.449}

Background Idiopathic scoliosis accounts for approximately $80 \%$ of scoliosis cases and distorts the thoracic cavity during lung development causing a restrictive lung disease. We have recently shown that each $10^{\circ}$ of curve accounts for a fall of approximately $4 \%$ in $\mathrm{FEV}_{1} /$ FVC. $^{1}$ However, little is known about whether spinal fusion improves lung function in these patients.

We performed follow up spirometry on 18 children who had undergone spinal fusion (age 11-16) and compared this to pre-operative values.

Aims To investigate the change in FVC and $\mathrm{FEV}_{1}$ before and after surgery using spirometry, and the relationship between this and the age at surgery and degree of curvature at that time.

Methods Eighteen patients who had previously undergone surgery (aged 11-16) were studied. Current height and arm span (to allow comparison with pre-operative measurements) were measured and spirometry was performed by the lung-function department (who had also undertaken pre-operative measurements). Pre and post-operative data were compared as absolute measures, and as Z-scores, using parametric statistical methods.

Results Contrary to previous reports, there was an increase in lung function in most patients following surgery but this was much more apparent in younger children and in those with a greater Cobb angle. Improvement in $\mathrm{FEV}_{1}$ was statistically correlated with Cobb angle at surgery (Pearson's coefficient $=0.513 \mathrm{p}=0.027$ ) although changes in FVC did not reach statistical significance (Pearson's $=0.42 \mathrm{p}=0.07$ ). Younger age at surgery was also significantly predictive of greater improvement for both $\mathrm{FEV}_{1}$ and FVC (Pearson's coefficient $=-0.77$ and -0.85 respectively $\mathrm{p}<0.001)$. Worse lung function preoperatively predicted greater improvement.

Conclusions Scoliosis surgery does seem to result in improved lung function, particularly $\mathrm{FEV}_{1}$, and this is greater with Cobb angles over $50^{\circ}$ and those with poorer lung function pre-operatively. At smaller angles there is little improvement in lung function. Younger patients may also see greater improvement.

\section{REFERENCE}

1. Saych L, et al. EAPS meeting. Barcelona 2014.

\section{G462(P) ASTHMANAUTS ARE GO! THE LAUNCH OF A NEW EFFECTIVE EDUCATIONAL RESOURCE FOR ASTHMA}

${ }^{1} \mathrm{D}$ Fenn, ${ }^{2} \mathrm{M}$ Fleet, ${ }^{3} \mathrm{~T}$ McLeod, ${ }^{1} \mathrm{C}$ Dat, ${ }^{1} \mathrm{~J}$ Moreiras. ${ }^{1}$ Paediatrics, Whittington Health NHS Trust, London, UK; ${ }^{2}$ Surgery, Scarborough General Hospital, Scarborough, UK; ${ }^{3}$ General Practice, Whipps Cross University Hospital, London, UK

\subsection{6/archdischild-2018-rcpch.450}

Aims Asthma is the most prevalent long term childhood disease and remains the commonest reason for urgent admissions to hospital in England. ${ }^{1}$ Providing effective asthma education is associated with reduced hospitalisations. ${ }^{2}$ 